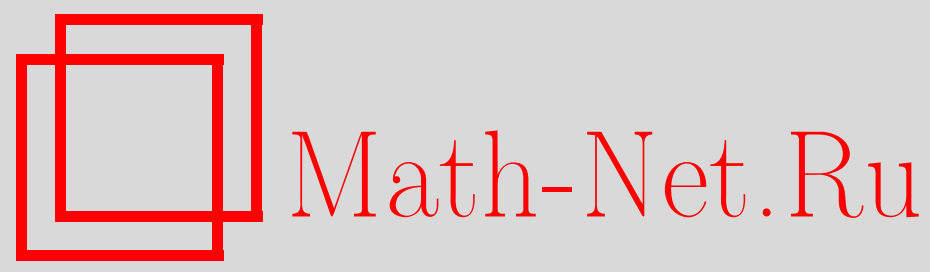

А. И. Шаповалов, Сопряженные интерполяционные задачи в пространствах целых функций, Матем. заметки, 2000, том 67, выпуск 4, 616-628

DOI: https://doi.org/10.4213/mzm877

Использование Общероссийского математического портала Math-Net.Ru подразумевает, что вы прочитали и согласны с пользовательским соглашением http://www.mathnet.ru/rus/agreement

Параметры загрузки:

IP : 18.209 .158 .208

26 апреля 2023 г., 13:31:36

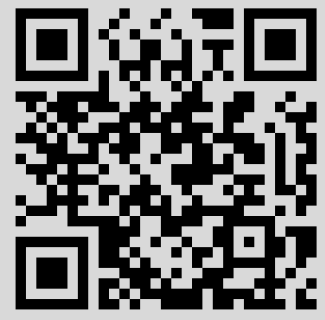




\section{СОПРЯЖЕННЫЕ ИНТЕРПОЛЯЦИОННЫЕ ЗАДАЧИ В ПРОСТРАНСТВАХ ЦЕЛЫХ ФУНКЦИЙ}

\section{А. И. Шаповалов}

Рассматривается понятие сопряженных интерполяционных задач на примере обобщения задачи и разложения целых функций в ряды Тейлора с переменным центром. Находятся радиусы существования, единственности и сходимости для таких задач. Находится связь между интерполяционными системами функционалов сопряженных друг с другом задач.

Библиография: 8 названий.

\section{§1. Введение и основные результаты}

Обозначим через $A_{R}(0<R<\infty)$ пространство регулярных в круге $\{|z|<R\}$ функций с топологией равномерной сходимости на компактах из $\{|z|<R\}$. Через $A_{0}(|z| \geqslant R)$ обозначим пространство функций, регулярных на $\{|z| \geqslant R\}$ и обрашающихся в нуль на бесконечности. Топология $A_{0}(|z| \geqslant R)$ задается равномерной сходимостью в какой-либо замкнутой области, содержащей $\{|z| \geqslant R\}$ строго внутри.

Всюду в дальнейшем, не оговаривая этого специально, через $m=\left\{m_{n}\right\}_{0}^{\infty}$ будем обозначать последовательность комплексных чисел, обладающих свойствами:

$$
m_{0}=1, \quad 0<\left|m_{n}\right|<\infty \quad(n=1,2, \ldots), \quad \lim _{n \rightarrow \infty}\left|m_{n}\right|^{1 / n}=\infty .
$$

С последовательностью $m$ будем связьвать целую функцию $\Phi(z)=\sum_{n=0}^{\infty} z^{n} / m_{n}$.

Обозначим через $A(\Phi, R)$ пространство целых функций $F(z)=\sum_{n=0}^{\infty} b_{n} z^{n} / m_{n}$ таких, что функции

$$
g(\Phi, F ; t)=\sum_{n=0}^{\infty} b_{n} t^{-n-1}
$$

принадлежат пространству $A_{0}(|z| \geqslant R)$.

Топология пространства $A(\Phi, R)$ индуцируется топологией сопряженного с ним пространства $A_{R}$. Свойства пространств $A(\Phi, R)$ хорошо изучены (см., например, [1]). Связь между $F(z)$ и $g(\Phi, F ; z)$ назьвается обобщенным преобразованием Бореля. Классическое преобразование Бореля соответствует функции $\Phi(z)=\exp (z)$.

Если $F(z) \in A(\Phi, R)$, то

$$
\varlimsup_{n \rightarrow \infty}\left|b_{n}\right|^{1 / n}<R .
$$


Пусть на некотором пространстве $A(\Phi, R)$ задана система линейных непрерьвных функционалов $\left\{L_{k}\right\}_{k=0}^{\infty}$. Хорошо известно (см., например, [2]), что их можно представить в виде

$$
\begin{gathered}
L_{k}[F]=\sum_{n=0}^{\infty} \frac{b_{n}}{m_{n}} l_{n}^{(k)}, \quad l_{n}^{(k)}=L_{k}\left[z^{n}\right], \quad n, k=0,1,2, \ldots, \\
\varlimsup_{n \rightarrow \infty}\left|\frac{l_{n}^{(k)}}{m_{n}}\right|^{1 / n} \leqslant \frac{1}{R} .
\end{gathered}
$$

Пусть

$$
l_{n}^{(k)}=0, k>n, \quad l_{n}^{(n)}=1, \quad n, k=0,1,2, \ldots
$$

Систему функционалов, удовлетворяющую условиям (3) и (4), будем назьвать интерполячионной системой функиионалов пространства $A(\Phi, R)$.

Интерполяционной системе функционалов $\left\{L_{k}\right\}_{k=0}^{\infty}$ поставим в соответствие систему полиномов $\left\{P_{n}(z)\right\}_{n=0}^{\infty}$ с помошью условий биортогональности:

$$
L_{k}\left[P_{n}\right]=\delta_{n, k} \quad\left(\delta_{n, k}=0, \quad n \neq k, \quad \delta_{k, k}=1, \quad n, k=0,1,2, \ldots\right) .
$$

Заметим, что условиями (5) каждый полином $P_{n}(z)$, степень которого равна в точности $n$, определяется единственным образом, и в силу (4) коэффициент при $z^{n}$ равен единище:

$$
P_{n}(z)=\sum_{k=0}^{n} P_{k}^{(n)} z^{k}
$$

Также и функционалы $L_{k}$ определяются через полиномы $P_{n}(z)$ с помощью (5) единственньм образом. Но не каждая система $\left\{P_{n}(z)\right\}_{n=0}^{\infty}$ определяет функционалы $\left\{L_{k}\right\}_{k=0}^{\infty}$ как линейные и непрерывные. Только в случае линейности и непрерьвности $\left\{L_{k}\right\}_{k=0}^{\infty}$ (т.е. вьполнения условия (3)) будем называть полиномы $\left\{P_{n}(z)\right\}_{n=0}^{\infty}$ интерполяиионной системой полиномов в соответствуюшем пространстве.

Интерполяционная задача состоит в восстановлении функции $F(z) \in A(\Phi, R)$ по заданной последовательности $\left\{L_{k}[F]\right\}_{k=0}^{\infty}$, где $\left\{L_{k}\right\}_{k=0}^{\infty}$ - интерполяционная система функционалов пространства $A(\Phi, R)$.

Другой способ постановки интерполяционной задачи состоит в задании интерполяционной системы полиномов $\left\{P_{n}(z)\right\}_{n=0}^{\infty}$.

Поставим произвольной функции $F(z) \in A(\Phi, r)(r>0)$ в соответствие формальный ряд

$$
F(z) \mapsto \sum_{n=0}^{\infty} L_{n}[F] P_{n}(z)
$$

который называется интерполячионным рядом функции $F(z)$.

Пространство $A(\Phi, R)(0<R \leqslant r)$ такое, что любая функция $F(z) \in A(\Phi, R)$ представляется сходящимся в топологии $A(\Phi, R)$ рядом $(6)$, назовем пространством сходимости интерполящионной задачи.

Пространство $A(\Phi, R)(R>0)$ такое, что условия $F(z) \in A(\Phi, R)$ и $L_{n}[F]=0$ $(n=0,1,2, \ldots)$ влекут за собой тождество $F(z)=0$, назьвается пространством единственности интерполяционной задачи.

Очевидно, что если $A(\Phi, R)$ является пространством сходимости, то оно представляет собой пространство единственности. 
Пусть $A=\left(a_{n, k}\right)_{n, k=0}^{\infty}$ - нижнетреугольная матрица с единицами на главной диагонали.

Матрица $A$ может задавать в каких-либо пространствах одновременно две интерполяционных задачи, одна из которых получается, если положить $a_{n, k}=l_{n}^{(k)}(n, k=0,1$, $2, \ldots$ ) , т.е. задать интерполяционную систему функционалов, а вторая, - если положить $a_{n, k}=P_{k}^{(n)}$, т.е. задать интерполяционную систему полиномов. Обозначим эти задачи соответственно $\omega$ и $\widetilde{\omega}$ и назовем сопряженными относительно матрицы $A$.

Матрица $B=\left(b_{n, k}\right)_{n, k=0}^{\infty}$, обратная к $A$, тоже будет нижнетреугольной с единицами на главной диагонали. Система интерполяционных полиномов задачи $\omega$ определяется равенствами $P_{k}^{(n)}=b_{n, k}(n, k=0,1,2, \ldots)$, а система интерполяционных функционалов задачи $\widetilde{\omega}$ определяется равенствами $l_{n}^{(k)}=b_{n, k}$.

Всюду в дальнейшем через $\beta=\left\{\beta_{n}\right\}_{n=0}^{\infty}$ будем обозначать последовательность конечных комплексных чисел таких, что

$$
\beta_{0}=1, \quad \sum_{n=1}^{\infty}\left|\beta_{n}\right|>0, \quad \lim _{n \rightarrow \infty}\left|\beta_{n}\right|^{1 / n}=0 .
$$

Определим класс $A_{m}^{\beta}(\Lambda)$ матриц $A(\lambda)$ таких, что

$$
a_{n, k}=\beta_{n-k} \frac{m_{n}}{m_{k}} \lambda_{n}^{n-k}, \quad n \geqslant k, \quad a_{n, k}=0, \quad n<k \quad(n, k=0,1,2, \ldots),
$$

где последовательность комплексных чисел $\lambda=\left\{\lambda_{n}\right\}_{n=0}^{\infty} \in \Lambda=\left\{\lambda:\left|\lambda_{n}\right| \leqslant 1, n=0,1\right.$, $2, \ldots\}$.

Через $B_{m}^{\beta}(\Lambda)$ обозначим класс матриц $B(\lambda)$, обратньх к $A(\lambda) \in A_{m}^{\beta}(\Lambda)$.

Каждый класс матриц $A_{m}^{\beta}(\Lambda)$ определяет два класса интерполяционных задач $\Omega_{m}^{\beta}(\Lambda)$ и $\widetilde{\Omega}_{m}^{\beta}(\Lambda)$, которые представляют собой множества интерполяционных задач $\omega=\omega(\lambda)$ и $\widetilde{\omega}=\widetilde{\omega}(\lambda), \lambda \in \Lambda$, сопряженных относительно матриц $A$, принадлежащих классу $A_{m}^{\beta}(\Lambda)$. В следуюших определениях символом $\Omega$ будем обозначать один из двух введенных вьше классов.

ОПРЕДЕЛЕНИЕ 1. Радиусом существования $R(\Omega, \Phi)$ класса $\Omega$ назовем наибольшее из чисел $R>0$ таких, что в пространстве $A(\Phi, R)$ существует интерполяционная система функционалов каждой задачи из класса $\Omega$.

ОПРЕДЕЛЕНИЕ 2. Радиусом единственности $E(\Omega, \Phi)$ класса $\Omega$ назовем наибольшее из чисел $R(R \leqslant R(\Omega, \Phi))$ таких, что пространство $A(\Phi, R)$ является пространством единственности каждой задачи из класса $\Omega$.

ОПРЕДЕЛЕНИЕ 3. Радиусом сходимости $S(\Omega, \Phi)$ класса $\Omega$ назовем наибольшее из чисел $R(R \leqslant R(\Omega, \Phi))$ таких, что пространство $A(\Phi, R)$ является пространством сходимости каждой задачи из класса $\Omega$.

ЗАмЕчАниЕ 1. В дальнейшем будет показано, что в определениях 1-3 действительно можно говорить о наибольшем $R$, а не только о точных верхних гранях.

ЗАмечаниЕ 2 . Очевидно, что $S(\Omega, \Phi) \leqslant E(\Omega, \Phi) \leqslant R(\Omega, \Phi)$.

Пусть матрица $A(\lambda)$ принадлежит классу $A_{m}^{\beta}(\Lambda)$. Тогда для взаимно-обратной матрицы $B(\lambda)$ справедливо равенство

$$
\sum_{i=k}^{n} a_{n, i} b_{i, k}=\delta_{n, k} .
$$


Отсюда, используя (7), получаем

$$
\frac{m_{k}}{m_{n}} b_{n, k}=-\sum_{i=0}^{n-k-1} \beta_{n-k-i} \lambda_{n}^{n-k-i} \frac{m_{k}}{m_{i+k}} b_{i+k, k}, \quad b_{k, k}=1, \quad \lambda \in \Lambda .
$$

Из (9) следует, что величина $b_{n, k}$ зависит лиш от $n-k$ чисел $\lambda_{k+1}, \lambda_{k+2}, \ldots \lambda_{n}$ и величина $m_{k} b_{n, k} / m_{n}$ не зависит от последовательности $m$.

Для любой матрицы $B(\lambda) \in B_{m}^{\beta}(\Lambda)$ определим последовательность $\gamma=\left\{\gamma_{n}\right\}_{n=0}^{\infty} \mathrm{c}$ помощью равенств

$$
\frac{1}{\left|m_{n-k}\right|} \max _{\lambda \in \Lambda}\left|b_{n-k, 0}\right|=\gamma_{n-k} \quad(n \geqslant k, \quad n, k=0,1,2, \ldots) .
$$

Последовательность $\gamma$ зависит лишь от $\beta$ и не зависит от $m$.

TEOPEMA 1. Cуществует

$$
\lim _{n \rightarrow \infty} \gamma_{n}^{-1 / n}=: W_{\beta}
$$

для которого справедливы соотношения

$$
\begin{gathered}
W_{\beta}=\inf _{n \geqslant 1} \gamma_{n}^{-1 / n}, \\
r_{|\beta|} \leqslant W_{\beta} \leqslant \inf _{|q|=1} r_{\beta}(q),
\end{gathered}
$$

где $r_{|\beta|}-$ единственный на интервале $(0,+\infty)$ корень иелой функции

$$
F_{|\beta|}(z)=1-\sum_{n=1}^{\infty}\left|\beta_{n}\right| z^{n}
$$

a $r_{\beta}(q)(q-$ - комплексное число такое, что $|q|=1)$ - расстояние от начала координат до ближайшего нуля иелой функиии

$$
F_{\beta, q}(z)=\sum_{n=0}^{\infty} \beta_{n} q^{(n(n+1)) / 2} z^{n}
$$

ТеОРема 2. Справедливы равенства

$$
\begin{gathered}
R\left(\Omega_{m}^{\beta}(\Lambda), \Phi\right)=\infty \\
R\left(\widetilde{\Omega}_{m}^{\beta}(\Lambda), \Phi\right)=W_{\beta}, \\
S\left(\Omega_{m}^{\beta}(\Lambda), \Phi\right)=E\left(\Omega_{m}^{\beta}(\Lambda), \Phi\right)=W_{\beta}, \\
S\left(\widetilde{\Omega}_{m}^{\beta}(\Lambda), \Phi\right)=E\left(\widetilde{\Omega}_{m}^{\beta}(\Lambda), \Phi\right)=W_{\beta} .
\end{gathered}
$$


ТЕОремА 3. Пусть $\left\{L_{n}\right\}_{n=0}^{\infty} u\left\{P_{n}(z)\right\}_{n=0}^{\infty}-$ интерполяиионные системы функuионалов и полиномов произвольной задачи $\omega(\lambda) \in \Omega_{m}^{\beta}(\Lambda), a\left\{V_{n}\right\}_{n=0}^{\infty} u\left\{G_{n}(z)\right\}_{n=0}^{\infty}$ интерполячионные системы функиионалов и полиномов сопряженной $\kappa \omega(\lambda)$ задачи $\widetilde{\omega}(\lambda)$. Тогда справедливы следующие утвержсдения.

1) Если иелая функиия $F(z)=\sum_{n=0}^{\infty} d_{n} z^{n}$ принадлежит пространству $A\left(\Phi, W_{\beta}\right)$, mо $L_{k}[F]=f^{(k)}(0) / k !(k=0,1,2, \ldots)$, где целая функиия $f(z)=\sum_{n=0}^{\infty} d_{n} G_{n}(z) n p u$ надлежит пространству $A\left(\Phi, W_{\beta}\right)$.

2) Если иелая функиия $f(z)=\sum_{n=0}^{\infty} d_{n} z^{n}$ принадлежит пространству $A\left(\Phi, W_{\beta}\right)$, mо $V_{k}[f]=F^{(k)}(0) / k !(k=0,1,2, \ldots)$, где иелая функиия $F(z)=\sum_{n=0}^{\infty} d_{n} P_{n}(z)$ nринадлежит пространству $A\left(\Phi, W_{\beta}\right)$.

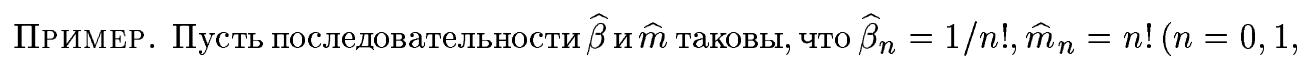
$2, \ldots)$. В этом случае функция $\Phi(z) \equiv \exp (z)$. Из $(7)$ следует, что

$$
a_{n, k}=\frac{n !}{(n-k) ! k !} \lambda_{n}^{n-k} \quad(n \geqslant k)
$$

Тогда интерполяционная система полиномов задачи $\widetilde{\omega}(\lambda) \in \widetilde{\Omega}_{\widehat{m}}^{\widehat{\beta}}(\Lambda)$ такова, что

$$
P_{n}(z)=\sum_{k=0}^{n} \frac{n !}{(n-k) ! k !} \lambda_{n}^{n-k} z^{k}=\left(z+\lambda_{n}\right)^{n}
$$

Полиномы вида (20) называются полиномами Тейлора с переменным центром. Разложение целых функций в ряды по таким полиномам изучалось многими авторами. В частности, Ю.А. Казьминым в [3] доказано, что $S\left(\widetilde{\Omega}_{\widehat{m}}^{\widehat{\beta}}(\Lambda), \exp \right)=W$, где $W$ - известная константа Уиттекера. Значит, как следует из теоремы 2 , в нашем случае $W_{\widehat{\beta}}=W$.

Найдем явньй вид интерполяционной системы функционалов этой задачи. Из (4) видно, что $L_{k}\left[z^{n}\right]=0, n<k$. Из (9) и (20) следует, что при $n \geqslant k$

$$
\begin{aligned}
L_{k}\left[z^{n}\right] & =b_{n, k}=-\sum_{i=k}^{n-1} \frac{\lambda_{n}^{n-i}}{(n-i) !} \frac{n !}{i !} b_{i, k}=\int_{\lambda_{n}}^{0} \sum_{i=k}^{n-1} \frac{z_{1}^{n-i-1}}{(n-i-1) !} \frac{n !}{i !} b_{i, k} d z_{1} \\
& =\int_{\lambda_{n}}^{0} d z_{1}\left(\sum_{i=k}^{n-2} \frac{z_{1}^{n-i-1}}{(n-i-1) !} \frac{n !}{i !} b_{i, k}+\frac{n !}{(n-1) !} b_{n-1, k}\right) \\
& =\int_{\lambda_{n}}^{0} d z_{1}\left(\sum_{i=k}^{n-2} \frac{z_{1}^{n-i-1}}{(n-i-1) !} \frac{n !}{i !} b_{i, k}-\sum_{i=k}^{n-2} \frac{\lambda_{n-1}^{n-i-1}}{(n-i-1) !} \frac{n !}{i !} b_{i, k}\right) \\
& =\int_{\lambda_{n}}^{0} d z_{1} \int_{\lambda_{n-1}}^{z_{1}} d z_{2}\left(\sum_{i=k}^{n-2} \frac{z_{2}^{n-i-2}}{(n-i-2) !} \frac{n !}{i !} b_{i, k}\right)=\cdots \\
& =\frac{n !}{k !} \int_{\lambda_{n}}^{0} d z_{1} \int_{\lambda_{n-1}}^{z_{1}} d z_{2} \ldots \int_{\lambda_{k+1}}^{z_{n-k-1}} d z_{n-k} .
\end{aligned}
$$




\section{§2. Доказательства теорем}

Для доказательства теорем 1-3 нам понадобиться ряд лемм.

Лемма 1. Пусть матрииа $A(\lambda) \in A_{m}^{\beta}(\Lambda)$ такова, что

$$
\lambda_{n}=q^{n} \quad(n=0,1,2, \ldots),
$$

где комплексное число $q:|q|=1$. Тогда для обратной матрищъ $B(\lambda)$ справедливо

$$
b_{n, k}=c_{n-k} \frac{m_{n}}{m_{k}} q^{n(n-1) / 2-k(k-1) / 2}, \quad n \geqslant k, \quad n, k=0,1,2, \ldots,
$$

где $\left\{c_{n}\right\}_{n=0}^{\infty}-$ последовательность коэффиииентов разлохения в ряд по степеням $z$ функиии $1 / F_{\beta, q}(z)=\sum_{n=0}^{\infty} c_{n} z^{n}$ (функиия $F_{\beta, q}(z)$, а также число $r_{\beta}(q)$ определены в теореме 1). Поэтому $c_{0}=1 u$

$$
\varlimsup_{n \rightarrow \infty}\left|c_{n}\right|^{1 / n}=\frac{1}{r_{\beta}(q)}
$$

Eсли жсе не существует нулей $F_{\beta, q}(z)$, mо $\varlimsup_{n \rightarrow \infty}\left|c_{n}\right|^{1 / n}=0$.

ДокАЗАТЕЛЬСТво. Из (9) и (21) получаем

$$
\frac{m_{k}}{m_{n}} b_{n, k}=-\sum_{i=0}^{n-k-1} \beta_{n-k-i} \frac{m_{k}}{m_{i+k}} q^{n(n-i-k)} b_{i+k, k}, \quad b_{k, k}=1 .
$$

Полагая $n=s+k$, перепишем последние рекуррентные соотношения в виде

$$
q^{-s(s+k)} \frac{m_{k}}{m_{s+k}} b_{s+k, k}=-\sum_{i=0}^{s-1} \beta_{s-i} q^{-i(s-i)} \frac{m_{k}}{m_{i+k}} q^{-i(i+k)} b_{i+k, k}, \quad b_{k, k}=1 .
$$

Отсюда видно, что величина $m_{k} q^{-i(i+k)} b_{i+k, k} / m_{i+k}$ не зависит от $k$ и от последовательности $m$. Обозначим эту величину $r_{i}$. Тогда $(24)$ можно представить в виде

$$
r_{s}=-\sum_{i=0}^{s-1} \beta_{s-i} q^{-i(s-i)} r_{i}, \quad r_{0}=1
$$

С помощью (15) нетрудно видеть, что коэффициенты $c_{s}$ могут быть определены с помощью рекуррентных соотношений

$$
q^{-s(s+1) / 2} c_{s}=-\sum_{i=0}^{s-1} \beta_{s-i} q^{-i(s-i)} c_{i} q^{-i(i+1) / 2}, \quad c_{0}=1
$$

Сравнивая полученное и (25), получаем

$$
q^{-s(s+1) / 2} c_{s}=r_{s}=\frac{m_{k}}{m_{s+k}} q^{-s(s+k)} b_{s+k, k} \quad(s=0,1,2, \ldots) .
$$

Отсюда, полагая $s=n-k$, получаем (22). Лемма доказана. 
ЛЕмма 2. Для любой матрииъ $B \in B_{m}^{\beta}(\Lambda)$ справедливо

$$
\left|b_{n, k}\right| \leqslant \mu_{n-k}\left|\frac{m_{n}}{m_{k}}\right| \quad(n \geqslant k, n, k=0,1,2, \ldots),
$$

где $\left\{\mu_{n}\right\}_{n=0}^{\infty}-$ последовательность полохительных чисел $\left(\mu_{0}=1\right)$, которье являются коэффициентами разложсения в ряд по степеням z функиии $1 / F_{|\beta|}(z)\left(F_{|\beta|}(z)\right.$, а также $r_{|\beta|}$ определены в теореме 1). Отсюда, в частности, следует, что

$$
\varlimsup_{n \rightarrow \infty} \mu_{n}^{1 / n}=\frac{1}{r_{|\beta|}} .
$$

ДоКАЗАТЕЛЬСТво. С помощью (9) получаем

$$
\left|\frac{m_{k}}{m_{n}}\right|\left|b_{n, k}\right| \leqslant \sum_{i=0}^{n-k-1}\left|\beta_{n-k-i}\right|\left|\frac{m_{k}}{m_{i+k}}\right|\left|b_{i+k, k}\right|, \quad b_{k, k}=1 .
$$

Отсюда следует (26), где полагаем

$$
\mu_{n-k}=\sum_{i=0}^{n-k-1}\left|\beta_{n-k-i}\right| \mu_{i}, \quad \mu_{0}=1
$$

Из (28) и (14) видно, что утверждения леммы доказаны.

ЗАмЕчАниЕ 3. Существует континуум матриц, для которых в (26) имеет место знак равенства. Чтобы показать это, для произвольного $q$ такого, что $|q|=1$, определим новую последовательность $\widetilde{\beta}=\widetilde{\beta}(q)$ :

$$
\widetilde{\beta}_{0}=\beta_{0}, \quad \widetilde{\beta}_{n}=-\left|\beta_{n}\right| q^{-n(n+1) / 2}, \quad n \geqslant 1 .
$$

Из (29) следует (в терминах лемм 1,2$)$, что $F_{\widetilde{\beta}, q}(z) \equiv F_{|\beta|}(z)$, и поэтому $c_{n}=\mu_{n}$ $(n=0,1,2, \ldots)$ и $r_{|\beta|}=r_{\tilde{\beta}}(q)$. Следовательно, для этого случая в $(26)$ знак равенства.

Лемма 3. Для любой матрицы $B \in B_{m}^{\beta}(\Lambda)$ справедливы равенства

$$
\left|\frac{m_{k}}{m_{n}}\right| \max _{\lambda \in \Lambda}\left|b_{n, k}\right|=\left|\frac{1}{m_{n-k}}\right| \max _{\lambda \in \Lambda}\left|b_{n-k, 0}\right|=\gamma_{n-k} \quad(n \geqslant k) .
$$

ДокАЗАтЕльСтво. Равенство (30) следует из (9) и принципа максимума модуля, если заметить, что $b_{n, k}$ и $b_{n-k, 0}$ зависят от одинакового количества переменных из последовательности $\lambda$, которые отличаются лишь нумерацией.

ЛЕмма 4. Для чисел $\gamma_{i}$, определенных в (10), справедливы соотношения

$$
\gamma_{n} \geqslant \gamma_{k} \gamma_{n-k} \quad(n \geqslant 1, \quad 1 \leqslant k \leqslant n)
$$


ДокАЗАТЕЛЬСТво. Индукцией по $k$ докажем формулу

$$
b_{n, 0}=b_{k, 0} b_{n, k}-\sum_{i=k}^{n-1} b_{n, i+1} \sum_{j=0}^{k-1} b_{j, 0} a_{i+1, j} .
$$

Из свойства взаимной обратности матриц $A(\lambda)$ и $B(\lambda)$ следует, что числа $b_{n, s}$ можно определять с помошь равенств

$$
b_{s, s}=1, \quad b_{n, s}=-\sum_{i=0}^{n-s-1} b_{n, s+i+1} a_{s+i+1, s} .
$$

Полагая в (33) $s=0$, получаем

$$
b_{0,0}=1, \quad b_{n, 0}=-\sum_{i=1}^{n-1} b_{n, i+1} a_{i+1,0}-b_{n, 1} a_{1,0} .
$$

Полагая в (8) $k=0$, получаем

$$
b_{n, 0}=-\sum_{i=0}^{n-1} b_{i, 0} a_{n, i} .
$$

Из (35) следует, что $b_{1,0}=-a_{1,0}$, и, подставляя это в (34), получаем, что формула (32) при $k=1$ верна. Предположим, что (32) верна для $k=p-1(p \geqslant 2)$ и покажем, что она верна тогда и для $k=p$. Выразим $b_{n, p-1}$ из (33) и подставим в формулу (32), написанную для $k=p-1$. Мы получим

$$
b_{n, 0}=b_{p-1,0}\left(-b_{n, p} a_{p, p-1}-\sum_{i=1}^{n-p} b_{n, i+p} a_{i+p, p-1}\right)-\sum_{i=p-1}^{n-1} b_{n, i+1} \sum_{j=0}^{p-2} b_{j, 0} a_{i+1, j} .
$$

Преобразовьвая, получаем

$$
b_{n, 0}=-b_{n, p}\left(\sum_{j=0}^{p-2} b_{j, 0} a_{p, j}+b_{p-1,0} a_{p, p-1}\right)-\sum_{i=p}^{n-1} b_{n, i+1} \sum_{j=0}^{p-1} b_{j, 0} a_{i+1, j} .
$$

Замечая, что выражение в скобке в правой части (36) равно $-b_{p, 0}$ (это следует из (35)), получаем, что формула (32) справедлива для $k=p$.

Из рекурентных соотношений (9) видно, что числа $b_{n, s}$ можно рассматривать как однородные полиномы от $\lambda_{s+1}, \lambda_{s+2}, \ldots, \lambda_{n}$, т.е.

$$
\begin{gathered}
b_{n, s} \equiv b_{n, s}\left(\lambda_{s+1}, \ldots, \lambda_{n}\right), \\
b_{n, s}\left(t \lambda_{s+1}, \ldots, t \lambda_{n}\right)=t^{n-s} b_{n, s}\left(\lambda_{s+1}, \ldots, \lambda_{n}\right) \quad t \in \mathbb{C} .
\end{gathered}
$$

Воспользуемся свойством однородности и формулой (32):

$$
b_{n, 0}\left(\lambda_{1}, \ldots, \lambda_{k}, t \lambda_{k+1}, \ldots, t \lambda_{n}\right)=t^{n-k}\left[b_{k, 0}\left(\lambda_{1}, \ldots, \lambda_{k}\right) b_{n, k}\left(\lambda_{k+1}, \ldots, \lambda_{n}\right)+C_{k}(t)\right] \text {, }
$$

где $C_{k}(t)$ - многочлен степени $k$ по переменной $t$ такой, что $C_{k}(0)=0$. Далее имеeм $\max _{|t| \leqslant 1}\left|b_{n, 0}\left(\lambda_{1}, \ldots, \lambda_{k}, t \lambda_{k+1}, \ldots, t \lambda_{n}\right)\right| \geqslant\left|b_{k, 0}\left(\lambda_{1}, \ldots, \lambda_{k}\right)\right|\left|b_{n, k}\left(\lambda_{k+1}, \ldots, \lambda_{n}\right)\right|$. Поскольку величины $b_{k, 0}$ и $b_{n, k}$ не имеют общих аргументов, то, беря в обеих частях последнего неравенства максимум по последовательности $\lambda \in \Lambda$ и учитывая лемму 3 , получаем (31). Лемма доказана. 
ДОКАЗАТЕЛЬСТВО ТЕОРЕМЫ 1. Для произвольных натуральных $n$ и $k(n \geqslant 2,1 \leqslant$ $k \leqslant n)$ выберем натуральное $p$ так, чтобы $0 \leqslant n-p k=s<k$. Тогда с помошью (31) получаем неравенство

$$
\gamma_{n} \geqslant \gamma_{k}^{p} \gamma_{s}=\gamma_{k}^{n / k} \gamma_{k}^{-s / k} \gamma_{s}
$$

откуда следует, что

$$
\underline{\lim }_{n \rightarrow \infty} \gamma_{n}^{1 / n} \geqslant \gamma_{k}^{1 / k}, \quad k=1,2, \ldots
$$

Беря в неравенстве (37) верхний предел по $k \rightarrow \infty$, получаем, что существует предел $\lim _{n \rightarrow \infty} \gamma_{n}^{-1 / n}$, который обозначим $W_{\beta}$, т.е. справедливо (11). Из (37) следует и равенство (12). Очевидно, что $c_{n} \leqslant \gamma_{n} \leqslant \mu_{n}$ (последовательности $c_{n}$ и $\mu_{n}$ определены в леммах 1,2$)$, откуда с помошю равенств (23) и (27) получаем (13). Теорема доказана.

ЗАмЕчАНИЕ 4. Для любой последовательности $\beta$ можно с помошью формул (29) взять последовательность $\widetilde{\beta}$, для которой $W_{\widetilde{\beta}}=r_{|\beta|}$.

Лемма 5. Для того чтобы система функиионалов $\left\{L_{k}(t)\right\}_{k=0}^{\infty}$, удовлетворяющая условиям (4), была интерполячионной для произвольной интерполяционной задачи в пространстве $A(\Phi, R)(R>0)$, необходимо и достаточно, чтобь функu,uu

$$
f_{k}(t) \equiv L_{z}(\Phi(t z))=t^{k} \sum_{i=0}^{\infty} \frac{l_{i+k}^{(k)}}{m_{i+k}} t^{i}, \quad k \geqslant 0
$$

принадлежсали пространству $A_{R}$.

ДокАЗАТЕЛьСтво. Утверждение (38) равносильно (3). Лемма доказана.

ЗАмЕЧАнИЕ 5. Из работы [1] следует, что

1) полнота в пространстве $A_{R}$ системы функций $\left\{f_{k}(t)\right\}_{k=0}^{\infty}$ заданной интерполяционной задачи равносильна тому, что пространство $A(\Phi, R)$ является пространством единственности этой интерполяционной задачи;

2) базисность в пространстве $A_{R}$ системы функций $\left\{f_{k}(t)\right\}_{k=0}^{\infty}$ заданной интерполяционной задачи равносильна тому, что пространство $A(\Phi, R)$ является пространством сходимости этой интерполяционной задачи.

При доказательстве следующей леммы используется метод работы [4].

Лемма 6. Существует последовательность $\lambda^{*} \in \Lambda$ такая, ито

1) система функиий $\left\{f_{k}(z)\right\}_{k=0}^{\infty}$ задачи $\omega\left(\lambda^{*}\right) \in \Omega_{m}^{\beta}(\Lambda)$ не является полной в пространствах $A_{R}$ для любого $R>W_{\beta}$,

2) при некотором $k=0,1,2, \ldots$ справедливо равенство

$$
\varlimsup_{n \rightarrow \infty}\left|\frac{b_{n, k}^{*}}{m_{n}}\right|^{1 / n}=\frac{1}{W_{\beta}}
$$

əде $b_{n, k}^{*} \equiv b_{n, k}\left(\lambda_{k+1}^{*}, \ldots, \lambda_{n}^{*}\right)$. 
ДокАЗАтЕЛЬСтво. Рассмотрим в соответствии с леммой 5 систему функций

$$
f_{k}(z)=z^{k} \sum_{s=0}^{\infty} \frac{a_{k+s, k} z^{s}}{m_{k+s}}, \quad k \geqslant 0 .
$$

Из (39), (7), а также условий (М) и (В) видно, что функции $f_{k}(z)$ являются целыми и задают интерполяционную систему функционалов задачи $\omega(\lambda)$. Для функций $(39)$, учитывая условия (В), для любого $\rho \geqslant 0$ и любого $\lambda \in \Lambda$ справедливы неравенства

$$
\varlimsup_{k \rightarrow \infty}\left[m_{k} \max _{|z| \leqslant \rho}\left|f_{k}(z)\right|\right]^{1 / k} \leqslant \varlimsup_{k \rightarrow \infty}\left[\rho^{k} \sum_{0}^{\infty} \beta_{s} \rho^{s}\right]^{1 / k} \leqslant \rho .
$$

Воспользуемся рассуждениями Евграфова [5, с. 110-113].

Возьмем произвольное натуральное число $n$. Выберем числа $\lambda_{1}^{(n)}, \ldots, \lambda_{n}^{(n)}$ так, чтобы $\left|\lambda_{i}^{(n)}\right|=1(i=1, \ldots, n)$ и определенные с их помошью величины $b_{n, k}^{(n)} \equiv b_{n, k}\left(\lambda_{1}^{(n)}\right.$, $\left.\ldots, \lambda_{n}^{(n)}\right)$ удовлетворяли бы равенствам $\left|b_{n, 0}^{(n)}\right| /\left|m_{n}\right|=\gamma_{n}$. Если остальные $\lambda_{i}^{(n)}(i=$ $n+1, n+2, \ldots)$ положить произвольными, но с условием $\left|\lambda_{i}^{(n)}\right| \leqslant 1$, то определенные для такой последовательности $\lambda^{(n)} \in \Lambda$ интерполяционные функционалы задачи $\omega\left(\lambda^{(n)}\right)$ будем обозначать $L_{k}^{(n)}$, а элементы матрицы $A\left(\lambda^{(n)}\right)$ будем обозначать $a_{s, k}^{(n)}$.

Рассмотрим последовательность полиномов

$$
Q_{0}(z) \equiv 1, \quad Q_{n}(z)=\frac{1}{b_{n, 0}^{(n)}} \sum_{s=0}^{n} b_{n, s}^{(n)} z^{s}, \quad n \geqslant 1 .
$$

Из (41) видно, что $Q_{n}(0)=1(n \geqslant 0)$. Из (41) получаем

$$
L_{k}^{(n)}\left(Q_{n}\right)=\frac{1}{b_{n, 0}^{(n)}} \sum_{s=k}^{n} b_{n, s}^{(n)} a_{s, k}^{(n)}=0, \quad k=0,1, \ldots, n-1 .
$$

Используя лемму 4 , имеем

$$
\left|Q_{n}(z)\right| \leqslant \sum_{s=0}^{n} \frac{\gamma_{n-s}}{\gamma_{n}} \frac{|z|^{s}}{\left|m_{s}\right|} \leqslant \sum_{s=0}^{\infty} \frac{|z|^{s}}{\gamma_{s}\left|m_{s}\right|}, \quad n \geqslant 0 .
$$

Неравенства (42) показьвают, что последовательность $\left\{Q_{n}(z)\right\}_{n=0}^{\infty}$ является равномерно ограниченной, следовательно, из нее можно выбрать равномерно сходящуюся на каждом компакте подпоследовательность $\left\{Q_{n_{s}}(z)\right\}_{s=0}^{\infty}$. Ее предел обозначим через $Q(z)=$ $1+\sum_{i=1}^{\infty} q_{i} z^{i} \not \equiv 0$. Из построения видно, что $\left|q_{i}\right| \leqslant 1 /\left(\gamma_{i}\left|m_{i}\right|\right)$, следовательно, $Q(z) \in$ $A(\Phi, R), R>W_{\beta}$.

Если через $\lambda^{*}$ обозначить последовательность чисел $\lambda_{i}^{*}$, каждое из которых есть предельная точка последовательности $\lambda_{i}^{(n)}\left(n=n_{s}, s=1,2, \ldots\right),\left(\left|\lambda_{i}^{*}\right|=1, i=1,2, \ldots\right)$, то для интерполяционных функционалов $L_{n}^{*}$ задачи $\omega\left(\lambda^{*}\right)$ будет выполнено $L_{n}^{*}(Q)=0$, $n=0,1, \ldots$ Отсюда и из принципа двойственности, связьвающего полноту и единственность (см., например, [1]), заключаем, что утверждение 1) леммы верно.

Определим с помошью равенств

$$
\mathscr{L}_{n}^{*}\left(z^{k}\right)=m_{k} b_{n, k}^{*}, \quad k \leqslant n, \quad \mathscr{L}_{n}^{*}\left(z^{k}\right)=0, \quad k>n \quad(n, k=0,1, \ldots),
$$


систему функционалов. Из (43) и [2] следует, что $\left\{\mathscr{L}_{n}^{*}\right\}_{n=0}^{\infty}-$ система линейных и непрерьвных в $A_{R}$ функционалов при любом $R>0$.

Из (43) и (39) легко следует, что для функций $f_{k}(z) \equiv f_{k}\left(z, \lambda^{*}\right)$ задачи $\omega\left(\lambda^{*}\right)$, определенной с помошью матрицы $\left(a_{n, k}^{*}\right)_{n, k=0}^{\infty}$, справедливо

$$
\mathscr{L}_{n}^{*}\left(f_{k}\left(z, \lambda^{*}\right)\right)=\sum_{s=0}^{n-k} a_{k+s, k}^{*} b_{n, k+s}^{*}=\delta_{n, k},
$$

т.е. система $\left\{\mathscr{L}_{n}^{*}\right\}_{n=0}^{\infty}$ биортогональна с системой $\left\{f_{k}\left(z, \lambda^{*}\right)\right\}_{k=0}^{\infty}$. Поэтому $z^{k}$ можно поставить в соответствие формальньй ряд

$$
z^{k} \mapsto m_{k} \sum_{n=k}^{\infty} b_{n, k}^{*} f_{n}\left(z, \lambda^{*}\right)
$$

Из определения числа $W_{\beta}$ следует, что для любой матрицы $B(\lambda) \in B_{m}^{\beta}(\Lambda)$ для $k=0,1, \ldots$ справедливо неравенство $\varlimsup_{n \rightarrow \infty}\left|b_{n, k} / m_{n}\right|^{1 / n} \leqslant 1 / W_{\beta}$. Предположим, что утверждение 2) леммы неверно, а именно, существует число $R>W_{\beta}$, для которого при всех $k=0,1, \ldots$ и всех $\lambda \in \Lambda$ выполняются неравенства $\varlimsup_{n \rightarrow \infty}\left|b_{n, k} / m_{n}\right|^{1 / n} \leqslant 1 / R$. Из этого предположения и из (40), где $\rho$ выбираем из интервала $\left(W_{\beta}, R\right)$, получаем неравенство

$$
\varlimsup_{n \rightarrow \infty}\left[\left|b_{n, k}^{*}\right| \max _{|z| \leqslant \rho}\left|f_{n}\left(z, \lambda^{*}\right)\right|\right]^{1 / n} \leqslant \frac{\rho}{R}<1,
$$

которое показьвает, что ряд (44) равномерно сходится в круге $|z| \leqslant \rho$, а поскольку система $\left\{f_{n}\left(z, \lambda^{*}\right)\right\}_{n=0}^{\infty}$ является первой приведенной системой, то (см. [6]) этот ряд сходится именно к функции $z^{k}$, т.е. в (44) имеет место равенство и, следовательно, система $\left\{f_{n}\left(z, \lambda^{*}\right)\right\}_{n=0}^{\infty}$ полна в $A_{R}, R>W_{\beta}$, что невозможно. Лемма доказана.

Рассмотрим квазистепенную базисность систем функций $\left\{f_{n}(t) \equiv L_{n}(\Phi(t z))\right\}_{n=0}^{\infty}$ заданной интерполяционной задачи.

Система $\left\{f_{n}(z)\right\}_{n=0}^{\infty}$ называется квазистепенным базисом (КС-базисом) в $A_{R}$, $R>0$, если любая $f(z) \in A_{R}$ представляется (единственным образом) сходяшимся в топологии $A_{R}$ рядом $f(z)=\sum_{n=0}^{\infty} x_{n} f_{n}(z)$ таким, что $\varlimsup_{n \rightarrow \infty}\left|x_{n}\right|^{1 / n}<R$, и, обратно, если последовательность $\left\{x_{n}\right\}_{n=0}^{\infty}$ удовлетворяет предыдущему неравенству, то ряд $f(z)=\sum_{n=0}^{\infty} x_{n} f_{n}(z)$ сходится в топологии $A_{R}$.

Из определения видно, что системы функций мы должны нормировать.

ЛЕмма 7. Система функиий $\left\{m_{n} f_{n}(z)\right\}_{n=0}^{\infty}$ произвольной задачи $\omega(\lambda) \in \Omega_{m}^{\beta}(\Lambda)$ образует KС-базис в пространствах $A_{R}$ для любого $R \in\left(0, W_{\beta}\right]$.

ДокАЗАТЕЛЬСТВО. Найдем явный вид целых функций $m_{n} f_{n}(z)$. Из леммы 5 и $(7)$ следует, что

$$
m_{n} f_{n}(z)=z^{n} \sum_{s=0}^{\infty} \beta_{s} \lambda_{s+n}^{s} z^{s} .
$$

Единственной биортогональной с функциями (45) является система функций $g_{n}(t) / m_{n} \equiv$ $g\left(\Phi, P_{n}(z) ; t\right)$, где $P_{n}(z)$ - интерполяционные полиномы рассматриваемой задачи. 
Из (1) следует, что

$$
g_{n}(t)=\sum_{s=0}^{n} m_{s} b_{n, s} t^{-s-1} .
$$

Из (46) видно, что $g_{n}(t) \in A_{0}(|t| \geqslant \varepsilon)$ для для любого $\varepsilon>0$.

Воспользуемся критерием КС-базисности, данным в [7]. В нашем случае надо проверить два утверждения:

1) для любого $r \in(0, R)$

$$
\varlimsup_{n \rightarrow \infty}\left[\max _{|z| \leqslant r}\left|m_{n} f_{n}(z)\right|\right]^{1 / n}<R
$$

2) для каждого фиксированного $\rho \in(0, R)$ сушествует $\mu=\mu(\rho) \in(0, R)$ такое, что

$$
\varlimsup_{n \rightarrow \infty}\left[\max _{|t| \geqslant \mu}\left|\frac{g(t)}{m_{n}}\right|\right]^{1 / n} \leqslant \frac{1}{\rho} .
$$

Докажем первое утверждение. Из (45) и условий (В) следует, что

$$
\max _{|z| \leqslant r}\left|m_{n} f_{n}(z)\right| \leqslant r^{n} \sum_{0}^{\infty}\left|\beta_{s}\right| r^{s}=r^{n} B(r),
$$

где $B(r)$ есть константа, зависящая от $r$, а так как $r<R$, то 1) доказано.

Для доказательства второго утверждения положим $\mu(\rho)=\rho$. Тогда из (46) и определения чисел $\gamma_{s}$ следует, что

$$
\max _{|t| \geqslant \rho}\left|\frac{g_{n}(z)}{m_{n}}\right| \leqslant \frac{1}{\rho^{n+1}} \sum_{s=0}^{n} \gamma_{n-s} \rho^{n-s} \leqslant \frac{1}{\rho^{n+1}} \sum_{s=0}^{\infty} \gamma_{s} \rho^{s},
$$

а так как $\rho<W_{\beta}$, то ряд в последнем неравенстве сходится, откуда и следует 2$)$. Лемма доказана.

ЛЕмма 8. Система функиий $\left\{m_{n} f_{n}(z)\right\}_{n=0}^{\infty}$ произвольной задачи $\widetilde{\omega}(\lambda) \in \widetilde{\Omega}_{m}^{\beta}(\Lambda)$ образует КС-базис в пространствах $A_{R}$ для любого $R \in\left(0, W_{\beta}\right]$.

ДокАЗАТЕЛЬСтвО. Найдем явньй вид функций $m_{n} f_{n}(z)$. Из леммы 5 следует, что

$$
m_{n} f_{n}(z)=z^{n} \sum_{s=0}^{\infty} m_{n} b_{s+n, n} \frac{z^{s}}{m_{s+n}} .
$$

Из (49), определения числа $W_{\beta}$ и леммы 6 видно, что функции $f_{n}(z)$ являются регулярными в круге $|z|<W_{\beta}$. Единственной биортогональной с функциями (49) является система функций $g_{n}(t) / m_{n} \equiv g\left(\Phi, P_{n}(z) ; t\right)$, где $P_{n}(z)$ - интерполяционные полиномы задачи $\widetilde{\omega}(\lambda)$.

Из (1) и (7) следует, что

$$
\frac{g_{n}(t)}{m_{n}}=\sum_{s=0}^{n} \beta_{n-s} \lambda_{n}^{n-s} t^{-s-1}
$$

Из (50) видно, что $g_{n}(t) \in A_{0}(|t| \geqslant \varepsilon)$ для всех $\varepsilon>0$.

Воспользуемся критерием КС-базисности из [7], а именно, в нашем случае надо доказать утверждения (47) и (48).

Доказательство (47) следует из того факта, что рядв (49) сходится при $|z| \leqslant \rho \leqslant W_{\beta}$. Для доказательства (48) положим $\mu(\rho)=\rho$. Далее, при оценке нужного нам максимума модуля конечную сумму увеличим до бесконечной и, воспользовавшись условиями (В), получим утверждение (48). Лемма доказана. 
ДОКАЗАТЕЛЬСТВО ТЕОРЕмЫ 2. Утверждение (16) следует из (7), леммы 5 и условий (М) и (В). Для доказательства (17) нужно еще второе утверждение леммы 6 . Так как из квазистепенной базисности следует просто базисность, то из лемм $6,7,8$, замечания 2 , а также замечания 5 получаем утверждения (18) и (19) теоремы. Теорема доказана.

ЗАмЕчАнИЕ 6. Из работы [8] следует, что леммы 7, 8 позволяют утверждать, что соответствующие интерполящионные ряды сходятся в топологии более сильной, чем топология пространства $A(\Phi, R)$ для любых $R \leqslant W_{\beta}$.

ДОКАЗАТЕЛЬСТВО ТЕОРЕМЫ 3. 1). Используя обозначения теоремы, легко видим, что $d_{n}=V_{n}[f]$; поэтому из теоремы 2 заключаем, что функция $f(z)$ принадлежит пространству $A\left(\Phi, W_{\beta}\right)$. Далее, производя почленное дифференцирование ряда функции $f(z)$ и учитьвая, как связаны коэффициенты интерполяционных полиномов и функционалов сопряженных задач, мы получаем

$$
\frac{f^{(k)}(0)}{k !}=\sum_{n=k}^{\infty} d_{n} \frac{G_{n}^{(k)}(0)}{k !}=\sum_{n=k}^{\infty} d_{n} G_{k}^{(n)}=\sum_{n=k}^{\infty} d_{n} l_{n}^{(k)}=L_{k}[F] .
$$

Вторая часть теоремы доказывается аналогично. Теорема доказана.

\section{СПИСОК ЦИТИРОВАННОЙ ЛИТЕРАТУРЫ}

[1] Евграфов М. А. Основные понятия интерполяции целых функций. препринт 20. М.: Ин-т прикладной матем. АН СССР, 1975.

[2] Маркушевич А. И. О базисе в пространстве аналитических функций // Матем. сб. 1945. T. 17. № 2. C. 211-252.

[3] Казьмин Ю. А. Возмущенные многочлены Аппеля и ассоциированные с ними системы функций // Докл. АН СССР. 1985. Т. 282. №1. С. 19-22.

[4] Осколков В.А. О базисности некоторых полиномиальных систем в пространствах целых функций экспоненциального типа // Изв. РАН. Сер. матем. 1993. Т. 57. № 3. С. 179-191.

[5] Евграфов М. А. Интерполяционная задача Абеля-Гончарова. М.: Гостехиздат, 1954.

[6] Евграфов М. А. Метод близких систем в пространстве аналитических функций и его применения к интерполяции // Труды московского матем. общества. 1956. Т. 5. С. 89-201.

[7] Осколков В.А. Об одном критерии квазистепенного базиса и его применении // Матем. заметки. 1990. Т. 48. №6. С. $72-78$.

[8] Осколков В. А. Свойства функций, заданных значениями их линейных функционалов. Дисс. ․ д.ф.-м.н. М.: МГУ, 1994. 